Ann. Biol. anim. Bioch. Biophys., 1977, 17 (5 B), 897-904.

\title{
Influence du régime sur les quotients respiratoires chez le porc en croissance
}

par Geneviève CHARLET-LERY, Marie-Thérèse MOREL

Laboratoire de Physiologie de la Nutrition

I.N.R.A., 78350 jouy-en-josas

Summary. Influence of diet on respiratory quotients and fat deposition in growing pigs.

Shortlasting measurements of gaseous exchanges in total confinement repeated several times during the day were used to study variations in these exchanges and in the respiratory quotient (RQ) during $23 \mathrm{~h}$ after meal intake in growing pifs $(35-65 \mathrm{~kg})$.

The $Q R$ variations showed that lipogenesis took place during the first post-intake hours, whatever the protein concentration of the diet : nitrogen free, normal or high nitrogen level ( 25 p. $100 \mathrm{DM})$. In the last conditions values less than one $(0.85)$ were observed during lipogenesis.

Fat deposition $/ W^{0,75}$ was highly correlated with ingested carbohydrates/W0,75 $(r=+0.99 \mathrm{p}>0.01)$, and energy efficiency of fat deposition $\left(k_{f}\right)$ was 0.76 . Any decrease of the carbohydrate supply, compensated for by an increase in the protein supply, slows down lipogenesis in growing pigs.

\section{Introduction.}

De très nombreux travaux ont montré les liaisons qui existent, chez le porcen croissance, entre les caractéristiques quantitatives ef qualitatives des régimes et l'importance relative des dépôts protéiques et gras des carcasses obtenues. Or, chez ces animaux, l'apport énergétique de la ration étant surtout constitué de glucides la transformation métabolique de ces derniers en lipides corporels implique la formation d'importantes quantités de $\mathrm{CO}_{2}$.

Du fait de l'existence du pool carbonate de l'organisme susceptible de modifier momentanément la production de $\mathrm{CO}_{2}$ par rejet ou prélèvement, et donc le $Q R$, celui-ci est habituellement mesuré pendant des périodes relativement longues. Cependant, malgré cetfe cause d'erreurs, l'étude des modifications journalières que subit ce coefficient mesuré pendant des périodes inférieures à 30 minutes, nous a paru susceptible d'apporter des précisions sur la lipogenèse du porc.

Dans le cadre d'une recherche sur les dépenses prandiales et post-prandiales du porc en croissance, nous avons mis au point une méthode de mesure des échanges. gazeux de courte durée (Charlet-Lery, 1975). La nécessité d'une bonne adaptation de l'animal au milieu expérimental, la briéveté des mesures et leur précision nous 
ont conduit à utiliser une cage à métabolisme classique où le porc séjourne normalement, transformable en cage à confinement où la dilution des gaz respiratoires dans un espace limité permet une bonne précision analytique.

\section{Matériel et méthodes.}

Les porcs mâles castrés de race Large White $(30-60 \mathrm{~kg})$ reçoivent 2 repas par jour à $9 \mathrm{~h}$ et à $17 \mathrm{~h}$ sous forme semi-liquide ef certains repas du matin sont remplacés par une simple buvée. Admettant la similifude des conséquences des repas du matin et du soir, nos mesures avant, pendant et après le repas du matin ou la buvée qui le remplace permettent de suivre les échanges gazeux entre les $1^{\text {re }}$ et $23^{e}$ heures postprandiales.

Les mélanges alimentaires utilisés sont isoénergétiques ( $3000 \mathrm{kcal} E M$ environ). Leur apport de matière sèche est compris entre 65 et $120 \mathrm{~g} / \mathrm{Pkg}^{0,75}$ (tabl. 1). Leur concentration protéique (0-25 p.100MS) varie aux dépends des sources glucidiques $\left.{ }^{(}\right)$. L'adaptation des animaux à leur cage et aux mesures est de 3 semaines ef l'adaptation au régime de 10 jours minimum. La température ambiante est celle de la neutralité thermique de porcs non à jeun (18-210).

TABLEAU 1

Caractéristiques des régimes

\begin{tabular}{|c|c|c|c|c|c|c|c|c|}
\hline Animal & Période & $\begin{array}{l}\text { Poids } \\
\text { kg }\end{array}$ & $\begin{array}{c}\text { MS. i/repas } \\
g\end{array}$ & $\begin{array}{c}\text { EM/repas } \\
\text { kcal }\end{array}$ & $\begin{array}{r}N / M S \\
\text { P. } 100\end{array}$ & $\begin{array}{l}\text { MS. i } \\
/ P^{0,75} \\
g / j\end{array}$ & $\begin{array}{r}\text { Mad } \\
/ P^{0,75} \\
g / j\end{array}$ & $\begin{array}{c}\text { Glucides (7) } \\
\qquad / P^{0,75} \\
g / j\end{array}$ \\
\hline $\begin{array}{l}\text { Eta } \ldots \ldots \\
\text { Théta } \ldots \ldots \\
\text { Théta } \ldots \\
\text { lota } \ldots \\
\text { lota } \ldots \ldots \\
\text { lota ... . . }\end{array}$ & $\begin{array}{l}1 \\
3 \\
5 \\
1 \\
2 \\
3\end{array}$ & $\begin{array}{l}46,0 \\
39,6 \\
47,3 \\
37,3 \\
53,6 \\
63,4\end{array}$ & $\begin{array}{r}\left.910()^{1}\right) \\
514\left(^{2}\right) \\
927\left(^{3}\right) \\
775\left(^{4}\right) \\
1180\left(^{5}\right) \\
1302\left(^{6}\right)\end{array}$ & $\begin{array}{l}2740 \\
1491 \\
2688 \\
2382 \\
3620 \\
3949\end{array}$ & $\begin{array}{l}2,62 \\
4,65 \\
4,65 \\
1,82 \\
1,53 \\
0,02\end{array}$ & $\begin{array}{r}102,8 \\
65,1 \\
102,4 \\
102,6 \\
119,2 \\
115,7\end{array}$ & $\begin{array}{r}13,1 \\
15,4 \\
24,6 \\
10,1 \\
9,8 \\
0,0\end{array}$ & $\begin{array}{l}51 \\
23 \\
35 \\
51 \\
60 \\
93\end{array}$ \\
\hline
\end{tabular}

(1) Concentré protéique mixte à 7,76 N p. $100 \mathrm{MS}: 50$ p. 100 + orge : 50 p. 100 .

$\left({ }^{2}\right)$ et $\left({ }^{3}\right)$ même concentré protéique : 89 p. 100 + farine de baleine : 11 p. 100.

(4) Mélange protéiprive : 68 p. 100 + poudre de lait écrémé : 32 p. 100.

$\left({ }^{5}\right)$ Mélange protéiprive : 73 p. $100+$ poudre de lait écrémé : 27 p. 100.

$\left.{ }^{6}\right)$ Mélange protéiprive.

(7) Glucides : cérélose, saccharose, lactose, amidon, selon les régimes.

Les échanges gazeux sont mesurés le matin à jeun (Métabolisme de Base approchée ou $\mathrm{MBa}$ ), pendant le repas, puis 7 à 8 fois durant les 7 premières heures postprandiales uniquement lorsque les animaux sont couchés. Pour pallier les irrégularités d'horaire, on calcule par intrapolation les échanges gazeux à temps fixe $(30,60$,

(1) Cérélose et saccharose des régimes protéiprives, amidon des céréales, lactose de la poudre de lait écrémé. 
$90,120,180,240,300,360,420 \mathrm{mn}$ ) après le repas, le milieu du repas étant pris comme temps 0 . Quatre à 6 couples de jours (un jour-repas ef un jour-eau) sont étudiés par animal et par régime.

Les valeurs moyennes permettent donc de suivre l'évolution des échanges gazeux durant $23 \mathrm{~h}$ (fig. 1), de mesurer, par différence entre les phases post-repas et posteau, l'oxygène et l'anhydride carbonique correspondants à l'extra-chaleur durant les 30 et 420 e minutes post-prandiales (extra chaleur 30-420) (fig. 2), et de calculer par la formule de Brouwer les productions de chaleur correspondantes.

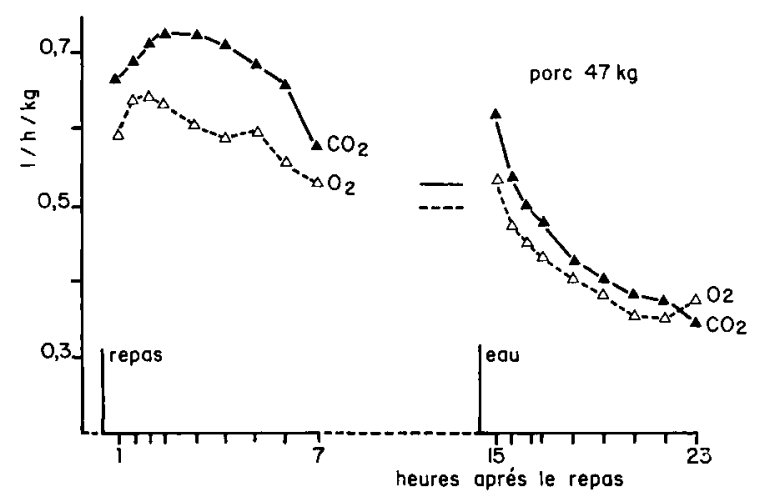

FIG. 1. - Cinétique des échanges respiratoires en fonction du temps écoulé depuis le repas.

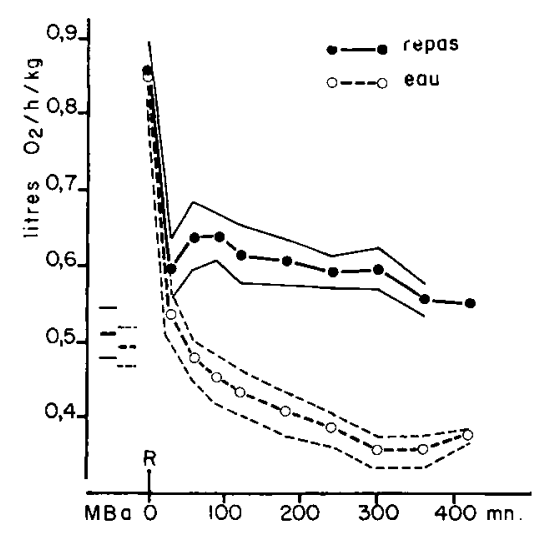

FIG. 2. - Consommation d'oxygène consécutive à l'ingestion d'un repas ou d'eau.

\section{Résultats.}

Quel que soit le régime, les valeurs intrapolées les plus élevées sont enregistrées pour l'oxygène au cours de la $2^{e}$ heure post-prandiale, pour l'anhydride carbonique au cours des 2 et $3 e$ heures, mais on constate de fortes irrégularités sur les données brutes.

Cependant durant les $7 \mathrm{~h}$ post-prandiales comme durant les $7 \mathrm{~h}$ post-eau, les consommations horaires $\mathrm{d}^{\prime} \mathrm{O}_{2}$ ne sont pas significativement différentes ou ne le sont que faiblement, il en est de même pour les productions de $\mathrm{CO}_{2}$. Ceci nous a permis d'étudier l'évolution des échanges gazeux à partir des données moyennes entre 1 ef 7,15 et 16,17 et $23 \mathrm{~h}$ de jeûne. Le tableau 2 donne l'ensemble des résultats.

Le calcul, par intrapolation à temps fixe, des valeurs post-repas ef post-eau entraîne en général une variabilité de leur moyenne plus faible que celle calculée à partir des valeurs brutes du MBa bien que pour ce dernier nous possédions un plus grand nombre de données.

La prolongation du jeûne abaisse ces valeurs plus rapidement pour $\mathrm{CO}_{2}$ (30 à 59 p. 100 de chute entre les intervalles $17-23$ et $1-7$ h) que pour $\mathrm{O}_{2}(20-41$ p. 100$)$. Les différences les plus fortes sont observées pour les régimes à teneur azotée normale ou inférieure à la normale, les plus faibles pour les régimes hyperazotés. 
II en résulte que les $Q R$ atteignent leurs valeurs maximales au cours des $7 \mathrm{~h}$ postprandiales et qu'ils sont significativement plus élevés après un repas qu'après ingestion d'eau.

\section{TABLEAU 2}

Evolution des échanges respiratoires moyens et des quotients respiratoires selon les animaux ef leur régime $(x \pm \mathrm{S} m)$

\begin{tabular}{|c|c|c|c|c|c|}
\hline \multicolumn{2}{|c|}{ Heures post-prandiales } & $\begin{array}{c}\text { Post-repas }(1) \\
1-7\end{array}$ & $\underset{15-16}{\mathrm{MBa}\left({ }^{2}\right)}$ & $\begin{array}{c}\text { Post-eau (1) } \\
17-23\end{array}$ & $\begin{array}{c}\text { Extra-chaleur } \\
30-420\end{array}$ \\
\hline \multirow[t]{3}{*}{ Eta 1} & $\mathrm{O}_{2}\left({ }^{3}\right)$ & $0,59+0,01$ & $0,50 \doteqdot 0,02$ & $0,40 \pm 0,02$ & $57,3\left({ }^{4}\right)$ \\
\hline & $\mathrm{CO}_{2}\left({ }^{3}\right)$ & $0,68 \pm 0,02$ & $0,53 \pm 0,02$ & $0,42 \pm 0,03$ & $80,5\left(^{4}\right)$ \\
\hline & $Q R$ & $1,14 \pm 0,02$ & $1,06 \pm 0,03$ & $1,06 \pm 0,03$ & $1,40^{\circ}$ \\
\hline \multirow[t]{3}{*}{ Theta 3} & $\mathrm{O}_{2}$ & $0,63 \pm 0,01$ & $0,62 \pm 0,02$ & $0,51 \pm 0,01$ & 33,4 \\
\hline & $\mathrm{CO}_{2}$ & $0,54 \pm 0,01$ & $0,48 \pm 0,01$ & $0,38 \pm 0,00$ & 40,4 \\
\hline & $Q R$ & $0,85 \pm 0,02$ & $0,79 \pm 0,02$ & $0,74 \pm 0,01$ & 1,21 \\
\hline \multirow[t]{3}{*}{ Theta 5} & $\mathrm{O}_{2}$ & $0,83+0,01$ & $0,66 \doteqdot 0,02$ & $0,59 \pm 0,01$ & 58,7 \\
\hline & $\mathrm{CO}_{2}$ & $0,72 \pm 0,01$ & $0,59 \pm 0,03$ & $0,47 \pm 0,02$ & 73,7 \\
\hline & $Q R^{2}$ & $0,87 \pm 0,01$ & $0,87 \pm 0,04$ & $0,74 \pm 0,02$ & 1,26 \\
\hline \multirow[t]{3}{*}{ lota 1} & $\mathrm{O}_{2}$ & $0,62 \pm 0,01$ & $0,47 \pm 0,02$ & $0,42 \pm 0,00$ & 48,3 \\
\hline & $\mathrm{CO}_{2}$ & $0,66 \pm 0,01$ & $0,47 \doteqdot 0,02$ & $0,33 \pm 0,01$ & 81,1 \\
\hline & $Q R$ & $1,07 \pm 0,01$ & $1,01 \pm 0,06$ & $0,78 \pm 0,02$ & 1,68 \\
\hline \multirow[t]{3}{*}{ lota 2} & & $0,60 \pm 0,02$ & $0,41 \pm 0,03$ & $0,36 \pm 0,01$ & 88,1 \\
\hline & $\mathrm{CO}_{2}$ & $0,72 \pm 0,02$ & $0,39 \pm 0,04$ & $0,29 \pm 0,00$ & 149,9 \\
\hline & $\mathrm{QR}^{-}$ & $1,19 \pm 0,01$ & $0,94 \pm 0,05$ & $0,82 \pm 0,01$ & 1,70 \\
\hline \multirow[t]{3}{*}{ lota 3} & $\mathrm{O}_{2}$ & $0,47 \pm 0,01$ & $0,36 \pm 0,01$ & $0,29 \pm 0,01$ & 73,2 \\
\hline & $\mathrm{CO}_{2}$ & $0,55 \nsubseteq 0,01$ & $0,33 \pm 0,02$ & $0,28 \pm 0,01$ & 111,7 \\
\hline & $Q R$ & $1,17 \div 0,02$ & $0,93 \pm 0,04$ & $0,95 \pm 0,01$ & 1,53 \\
\hline
\end{tabular}

(1) Moyenne de 7 données intrapolées aux temps 60,120,180, 240,300,360, $420 \mathrm{mn}$.

( $\left.{ }^{2}\right)$ Moyenne de 8 à 12 données brutes.

(3) Litres $/ \mathrm{kg} / \mathrm{h}$.

$\left({ }^{4}\right)$ Litres.

L'obtention séparée de $\mathrm{O}_{2}$ et de $\mathrm{CO}_{2}$ relatifs à l'extra-chaleur (30-420) permet de calculer le QR de cette extra-chaleur ; il est toujours nettement supérieur à l'unité : 1,20 à 1,70. Ces valeurs élevées, comme l'évolution des $Q R$ avec le temps, montrent l'intensité de la lipogenèse dans les premières heures qui suivent l'ingestion des repas, phénomène mis en évidence depuis fort longtemps (Wierzuchowski et Ling, 1925) mais qui, dans ce travail, est observé aussi avec des rations hyperprotéiques.

Le QR post-prandial de 1,17 et celui de l'extra-chaleur (30-420) de 1,53 obtenus avec le porc lota 3 recevant un aliment protéiprive à un niveau d'ingestion élevé (3949 kcal EM par repas) montrent l'existence d'une lipogenèse chez un tel animal, ce qui explique la constance de son poids malgré la perte de protéines endogènes d'environ $45 \mathrm{~g} / \mathrm{j}$. Aussi après 3 à 4 semaines d'un tel régime la carcasse du porc présente des caractères d'hyperadiposité et d'atrophie musculaire spectaculaires comme nous l'avons observé chez des animaux abatfus dans de telles conditions. Les résultats relatifs à cet animal sont éliminés des calculs ultérieurs. 
Influence des apports alimentaires sur le $Q R$.

En admettant que les divers glucides présents dans nos régimes (cérélose, saccharose, lactose, amidon de céréales essentiellement) sont utilisés d'une façon identique par le porc, nous avons pris leur somme en considération, soit 35 p. 100 pour les rations de Theta ef 50 p. 100 pour celles de Eta 1 ef lota 1 et 2.

Les QR (30-420) sont liés d'une façon significative à l'ingestion glucidique rapportée au poids métabolique : $r=+0,88, P<0,05$. II en est de même des QR postprandiaux. Par contre la liaison avec les matières azotées digestibles est négative $r=-0,77$ mais non significative.

Influence des apports alimentaires sur les dépôts lipidiques ef protéiques.

Pour apprécier l'importance des dépôts tissulaires, nous avons calculé l'énergie métabolisable disponible pour leur dépôt par différence selon l'équation

$E M$ totale - EM entretien = EM croissance

en admettant des besoins d'entretien pour un dépôt protéique nul égaux à $100 \mathrm{kcal} /$ $\mathrm{P}^{0,75}$ (Kielanowski et Kolarbinska, 1974 ; Thorbeck, 1974).

Les hypothèses suivantes:

- coût identique des dépôts pondéraux de protides et de lipides corporels, donc du dépôt énergétique total de $13 \mathrm{kcal} E M / \mathrm{g}$, soit des efficacité énergétiques respectives de 40 et 70 (Kielanowski et Kotarbinska, 1974),

- coefficients de digestibilité et de rétention protéique de nos animaux identiques à ceux préalablement mesurés sur d'autres animaux ou évalués par comparaison,

- rapport de 3,36 entre le dépôt protéique et les dépôts d'eau et de matières minérales qui constituent le reste du gain corporel selon les mêmes auteurs,

permettent de calculer successivement le dépôt pondéral total, le dépôt protéique et par différence le dépôt lipidique. La connaissance des dépôts d'eau et de matières minérales permet de calculer le gain de poids théorique de nos animaux au cours de chaque période (7-9 semaines).

Le tableau 3 résume ces calculs ef permet de constater que sauf pour le porc lota 3 soumis au régime protéiprive ef pour le porc Eła l, les gains réels sont peu différents des gains calculés.

Le dépôt lipidique est lié d'une façon significative à l'EM totale et plus encore à l'EM disponible pour la croissance $r=+0,97, P<0,01$.

La corrélation entre les glucides ingérés ef le dépôt pondéral, toutes données rapportées au poids métabolique, atteint $+0,99$ et est hautement significative $(P<0,01)$. La régression linéaire entre ces deux variables : $y=0,34 \times-5,8$ permet de retrouver un coefficient d'efficacité énergétique du dépôt lipidique $\left(K_{f}\right)$ de 76 p. 100 ce qui implique que cette valeur, égale aux valeurs habituellement trouvées 70-77 pour le dépôt lipidique, correspond essentiellement au coefficient de transformation des glucides en lipides.

Inversement et puisque la concentration en protéines de nos régimes varie en sens inverse de celle en glucides, le dépôt lipidique diminue lorsque l'apport azoté augmente, mais non significativement. 
TABLEAU 3

Evaluation des dépôts tissulaires et des gains de poids vif

\begin{tabular}{|c|c|c|c|c|c|c|c|}
\hline \multirow[b]{2}{*}{ Animal } & \multirow{2}{*}{$\begin{array}{c}\text { EM } \\
\text { croissance } \\
\text { kcal }\end{array}$} & \multicolumn{4}{|c|}{ Dépôt pondéral de tissu } & \multicolumn{2}{|c|}{ Gain } \\
\hline & & $\begin{array}{c}\text { total } \\
g\end{array}$ & $\begin{array}{c}\text { protéique } \\
\mathrm{g}\end{array}$ & $\begin{array}{c}\text { lipidique } \\
\mathrm{g}\end{array}$ & $\begin{array}{c}\text { eau }+ \\
\text { m.m. } \\
g\end{array}$ & $\begin{array}{c}\text { calculé } \\
\text { g }\end{array}$ & $\begin{array}{l}\text { observé } \\
\qquad g\end{array}$ \\
\hline $\begin{array}{l}\text { Eta } 1 \ldots \ldots \\
\text { Theta } 3 \ldots \ldots \\
\text { Theta } 5 \ldots \ldots \\
\text { lota } 1 \quad \ldots \ldots \\
\text { lota } 2 \ldots \ldots \\
\text { lota } 3 \ldots \ldots\end{array}$ & $\begin{array}{ll}3 & 710 \\
1 & 160 \\
3 & 296 \\
3 & 264 \\
5 & 250 \\
5 & 658\end{array}$ & $\begin{array}{r}285 \\
89 \\
253 \\
251 \\
404 \\
435\end{array}$ & $\begin{array}{r}92 \\
61 \\
133 \\
68 \\
98 \\
-45\end{array}$ & $\begin{array}{r}193 \\
28 \\
120 \\
183 \\
296 \\
480\end{array}$ & $\begin{array}{r}300 \\
199 \\
433 \\
222 \\
320 \\
-150\end{array}$ & $\begin{array}{l}585 \\
288 \\
686 \\
473 \\
712 \\
285\end{array}$ & $\begin{array}{l}360 \\
270 \\
830 \\
420 \\
610 \\
130\end{array}$ \\
\hline
\end{tabular}

Relation entre les dépôts lipidiques ef les $Q R$.

Les relations précédentes ont pour conséquence des liaisons étroites entre les $Q R$ et les dépôts lipidiques. Les coefficients de corrélation entre ces dépôt et les QR postprandiaux ou les QR de l'extra-chaleur (30-420) sont respectivement de $+0,92$ ef $+0,91$ au seuil de 5 p. 100, résultats qui sont en parfait accord avec les précédents.

Par conséquent, puisque l'augmentation de l'ingestion glucidique privilégie le dépôt lipidique par rapport au dépôt protéique, elle provoque une chute du rapport pondéral dépôt protéique/dépôt lipidique. Cette chute est exponentielle ce qui provoque une décroissance de même type des $Q R$ de l'extra chaleur lorsque ce rapport augmente (à la limite de la signification, $P<0,05)$ (fig. 3).

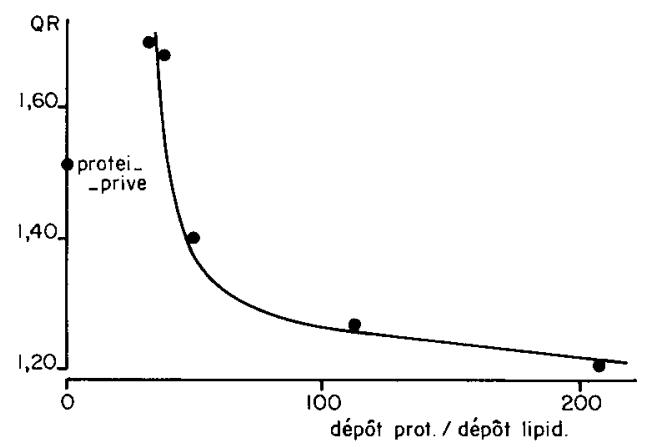

FIG. 3. - Evolution du quotient respiratoire de l'extra-chaleur (30-420) en fonction du rapporf dépôt protéique/dépôt lipidique.

\section{Discussion.}

L'utilisation des QR non corrigés est évidemment criticable mais la correction l'aurait été aussi puisque nous n'avons pas mesuré l'excrétion urinaire horaire d'azote. Cependant les $Q R$ non protéiques sont plus faibles que les $Q R$ non corrigés, et ce, 
d'autant plus que l'animal élimine plus d'azote urinaire ; aussi la différence entre régimes n'aurait pu que s'accentuer. Par contre, la différence entre les jours-repas et les jours-eau aurait été atténuée puisque l'animal élimine progressivement moins d'azote lorsque le jeûne s'accentue.

Les travaux de Cooke ef al. (1972) qui ont étudié le gain de poids et la composition des carcasses de porcs recevant des régimes apportant de 15 à 30 p. 100 de protéines et abattus à $58 \mathrm{~kg}$, confirment l'importance des dépôts lipidiques que nous avons calculés pour nos animaux. Cette lipogenèse, non négligeable chez les sujets recevant un excès protéique, apparaît en contradiction avec la faible valeur des $Q R$ post-prandiaux correspondants $(0,85$ et 0,87$)$. Cette contradiction explique les difficultés d'interprétation des $Q R$, valeur globale qui somme la totalité des réactions biochimiques ayant lieu à un moment donné dans l'organisme.

Les QR posi-prandiaux et surtout ceux d'extra-chaleur du porc lota 3 soumis au régime proféiprive sont légèrement plus bas que ceux des animaux recevant des régimes à faible concentration protéique $(1,53$ contre 1,68 et 1,70). Ce résultat est en accord avec une lipogenèse moins forte que ne le ferait supposer sa richesse en glucides solubles ( 80 p. 100$)$ : la carence totale de protéines expliquerait que l'animal n'ait pas pu effectuer les synthèses prévues.

Le comportement du porc pourrait expliquer les moments de la lipogenèse et son importance. En effet, les observations de Ruckebusch et Morel (1968) montrent que, chez le porc et dès l'âge de 3 mois, on observe toujours, consécutivement aux repas, de longues phases de décubitus et de sommeil, que son tonus musculaire structural disparaît partiellement lors du décubiłus et totalement dès les premières phases de sommeil et que au cours d'un nycthémère, il reste couché $20 \mathrm{~h}$ et endormi $13 \mathrm{~h}$.

\section{Conclusions.}

Chez le porc en croissance recevant des rations à faible teneur en lipides, l'évolution des $Q R$ dans le temps et l'importance des $Q R$ de l'extra-chaleur (30-420) montrent que la lipogenèse est surtout post-prandiale quelle que soit la concentration protéique du régime ; et ce, malgré des $Q R$ postprandiaux neftement inférieurs à $1(0,85-0,87)$ lorsque les régimes sont hyperazotés (24 p. 100).

La relation entre les glucides ingérés et le dépôt lipidique permet de calculer une efficacité énergétique de dépôt $\left(k_{f}\right)$ de 76 , coefficient classique qui confirme les hypothèses émises. Le comportement du porc expliquerait peut être l'intensité de sa lipogenèse et les moments où elle a lieu.

Réunion Groupe Développement INRA/Productions animales Thiverval-Grignon, 14-15 avril 1976.

\section{Références}

CHARLET-LERY G., 1975. Dépenses énergétiques prandiales et post-prandiales chez le porc en croissance. Thèse, Paris VI, PP. 129.

COOKE R., LODGE G. A., LEWI S., 1972. Influence of energy and protein concentration in the diet on the performance of growing pigs. I. Response to protein intake on a high-energy diet. Anim. Prod., 14, 35-46. 
KIELANOWSKI J., KOTARBINSKA M., 1974. Chemical composition and energy value of the liveweight gain of growing pigs. 6th Symp. Energy Metabolism of Farm Animals, 165-168.

RUCKEBUSCH Y., MOREL M. T., 1968. Etude polygraphique du sommeil chez le Porc. C. R. Soc. Biol., Paris, 162, 1346-1354.

THORBECK G., 1974. Energy metabolism in fasting pigs at different live weight as influenced by temperature. 6th Symp. Energy Metabolism of Farm Animals, 147-150. EAAP, publ. no 14, Universität Hohenheim, Stuttgart, B. R. D.

WIERZUCHOWSKI M., LING S. M., 1925. On fat production in a young hog. J. biol. Chem., 64, 697-707. 